\title{
Quantitative Ultrasound
}

National Cancer Institute

\section{Source}

National Cancer Institute. Quantitative Ultrasound. NCI Thesaurus. Code C150873.

A method of empirically measuring the velocity and attenuation of ultrasonic sound waves as they pass through tissue. 